\title{
Rapid, simple, low-cost smartphone-based fluorescence detection of Escherichia coli
}

\author{
Dante Rojas-Barboza ${ }^{1}$, Edward Park ${ }^{2}$, Rolfe Sassenfeld ${ }^{3}$, Jeremy Winder ${ }^{4}$, \\ Geoffrey B. Smith ${ }^{4}$, Delia Valles-Rosalles ${ }^{5}$, Efren Delgado ${ }^{1}$, Young Ho Park ${ }^{6 *}$ \\ (1. Family and Consumer Science Department, New Mexico State University, Las Cruces, NM 883003, USA; \\ 2. Biology Department, University of Pennsylvania, Philadelphia, PA 19104, USA; \\ 3. Electronics \& Computer Engineering Technology, New Mexico State University, Las Cruces, NM 88003, USA; \\ 4. Biology Department, New Mexico State University, Las Cruces, NM 88003, USA; \\ 5. Industrial Engineering Department, New Mexico State University, Las Cruces, NM 88003, USA; \\ 6. Mechanical Engineering Department, New Mexico State University, Las Cruces, NM 88003, USA)
}

\begin{abstract}
Food and waterborne diseases pose considerable public health threats even in highly industrialized parts of the world. Examples of these pathogens in food can be Escherichia coli O157: H7, Salmonella sp., and Listeria monocytogenes. Rapid, reliable detection of pathogens mitigates serious health problems and economic losses due to outbreaks and robust tests safeguard the food supply. In this study, a smartphone-based apparatus was employed to demonstrate quantitative detection of E. coli. To validate the applicability of the present smartphone-based fluorescence device, RNA was extracted from the $E$. coli $\mathrm{K}-12$ strain and amplified using two different primers (dnaK and $r p o A)$ via quantitative polymerase chain reaction (qPCR). Serial dilutions of RNA from 10 to $0.0001 \mathrm{ng} / \mu \mathrm{L}$ were prepared at the start of the PCR amplification and the PCR products were detected by CYBR Green1-based fluorescence. For a proof-of-concept test for the smartphone system, samples from these PCR products were then analyzed. The detection system employed a novel algorithm to analyze fluorescence signals and read changes in E. coli DNA concentration. The correlations between the fluorescence percentage and DNA concentrations were $R=0.945$ for the $d n a K$ primer and $R=0.893$ for the $r p o A$ primer, respectively. Utilizing this new fluorescent analysis technique resulted in comparable accuracy to the real-time PCR fluorescent signal detection. The key innovation of this approach was to combine efficient image processing encoded into a smartphone application with a low-cost 3-D printed device that allowed quantification of bacterial nucleic acid.
\end{abstract}

Keywords: smartphone, fluorescence, $E$. coli, low-cost, 3D-print

DOI: $10.25165 /$ j.ijabe.20211403.5865

Citation: Rojas-Barboza D, Park E, Sassenfeld R, Winder J, Smith G B, Valles-Rossalles D, et al. Rapid, simple, low-cost smartphone-based fluorescence detection of Escherichia coli. Int J Agric \& Biol Eng, 2021; 14(3): 189-193.

\section{Introduction}

The technical specifications of smartphones have advanced over the past decades in all aspects, including global positioning system (GPS), wireless technology (Bluetooth, Wi-Fi), powerful computing capability, and complementary metal oxide semiconductor (CMOS) digital camera sensor. These functionalities enable smartphone-based biosensing systems for point-of-care testing applications, which may allow for rapid, convenient diagnostic testing of pathogens. Particularly, a

Received date: 2020-05-21 Accepted date: 2020-09-27

Biographies: Dante Rojas-Barboza, Graduate Research Assistant, research interests: food microbiology, Email: danterb@nmsu.edu; Edward Park, Research Assistant, research interests: molecular biology, Email: edpark@ alumni.upenn.edu; Rolfe Sassenfeld, PhD, Associate Professor, research interests: Mobile app development, Email: rolfe@nmsu.edu; Jeremy Winder, Researcher, research interest: pathogen detection, Email: jwinder@nmsu.edu; Geoffrey Smith, PhD, Professor, research interests: pathgon detection, Email: gsmith@nmsu.edu. Delia Valles-Rosales, PhD, Associated Professor, research interests: data analytics, Email: dvalles@nmsu.edu; Efren Delgado, PhD, Associate Professor, research interests: food processing, Email: edelgad@ nmsu.edu.

*Corresponding author: Young Ho Park, PhD, Professor, research interests: sensing system development. Mechanical Engineering Department, New Mexico State University, Las Cruces, NM 88003, USA. Email: ypark@nmsu.edu. number of fluorescence imaging-based sensing techniques have been proposed using CMOS optical sensors in the smartphone. Fluorescent imaging-based detection methods show promise to utilize smartphones in analytical methods that apply a multiplexed fluorometric detection scheme $\mathrm{e}^{[1]}$. To label the biological target of interest, a fluorescent marker, which is able to bind the target molecule, needs to be introduced. The first smartphone-integrated visualization system that detected a single virus or bacterium was presented by Zhu et al. ${ }^{[2]}$. Their smartphone-based system offered a compact, light-weight solution for specific detection of the bacterium in low-resource settings. This group also achieved low-cost wide-field fluorescence imaging with an improved resolution by using parallel glass substrates instead of glass capillaries $^{[3]}$. Increased signal-to-noise ratio was demonstrated compared to traditional organic dyes by exploiting the high luminescent efficiency of quantum dots ${ }^{[4]}$.

An integrated system of fluorescent microscopy and imaging cytometry was developed by Zhu et al. ${ }^{[5]}$ on a smartphone using light-weight optofluidic attachment. This cell-phone based fluorescent imaging system allowed for the detection of Giardia lambia in drinking water. A similar concept was adopted by Ludwig et al. ${ }^{[6]}$ to develop a smartphone-based imaging system that could monitor anti-recombinant bovine somatotropin (rbST) antibodies; the proposed diagnostic tool detected fluorescent light 
emitted from quantum dot-coupled antibodies in milk extract. To reduce the cost of optical devices, Park et al. ${ }^{[7,8]}$ developed a portable device that detected Salmonella typhimurium on paper microfluidics; the device detected the bacteria by Mie scattering based on the image processed by the smartphone. In this method, the smartphone position and detection angle were optimized and only the ambient light source was used for image acquisition. Lee et al. ${ }^{[9]}$ presented a cell phone-based system for detecting lateral flow immunoassay (LFIA). Their system comprises an Android-based smartphone, a data analysis and image acquisition APP and an LFIA reader for diagnosis of aflatoxin-B1. In addition, a close-up lens and a white LED were used to enhance visual inspection of the fluorescent signal. Mora et al. ${ }^{[10]}$ demonstrated an analytical sensor containing genetically engineered bacteria that could fluoresce in reaction to the test object and demonstrated its diffusion behavior with a blue light source. This diagnostic analysis in combination with a smartphone allows inexperienced users to operate it. Recently, Priye et al. ${ }^{[11]}$ presented a portable device supplemented with a smartphone that can be used to detect Zika, dengue, and chikungunya virus using quenching of unincorporated amplification signal reporter technology. They employed the loop-mediated isothermal amplification (LAMP) technique that multiplies the target DNA sequence under isothermal conditions ${ }^{[12-14]}$.

In the present work, a rapid, cost-efficient device was developed by the authors to detect nucleic acid representing a food-borne pathogen (E. coli). The device comprised an LED light source, reflecting mirrors, an optical bandpass filter coupled with a smartphone platform, for quantitative analysis. The developed system was applied to E. coli as a proof-of-concept and was compared to traditional amplification with the use of iTaq Universal SYBR Green one-step kit ${ }^{[15]}$ utilizing RT PCR ${ }^{[16,17]}$.

\section{Materials and methods}

\subsection{Sample preparation}

Escherichia coli K-12 strain was grown in TGY broth (5 g tryptone, $3 \mathrm{~g}$ yeast extract, and $1 \mathrm{~g}$ glucose, per L) at $37^{\circ} \mathrm{C}$ for $18 \mathrm{~h}$.
E. coli RNA was extracted using gDNA elimination protocols based on RNeasy® Plus Micro Kit (QIAGEN) and the RNA was stored at $-80{ }^{\circ} \mathrm{C}$. The RNA template was converted to complementary DNA (cDNA), followed by 40 cycles of polymerase chain reaction. In this study, the $\mathrm{iTaq}^{\mathrm{TM}}$ Universal one-step PCR kit with SYBR Green I (Bio-Rad, Hercules, CA, USA) was used. Oligonucleotide primers for polymerase chain reaction amplification used in the present study were $r p o A$ and $d n a K$. The $r p o A$ gene is a control "housekeeping" gene expected to be expressed at a constant rate and the $d n a K$ gene is used as a representative of genes that would vary in its expression. Both genes have been used in previous work of the authors ${ }^{[18,19]}$. The NCBI GenBank Program was used to identify appropriate primers that would function to amplify these genes from the E. coli $\mathrm{K}-12$ genome. The PCR reaction volume was set up for $10 \mu \mathrm{L}$ using $2 \mu \mathrm{L}$ of template RNA. For negative control, RNA template was replaced by nuclease-free water. The PCR experiment was prepared on 96-well microplate which was sealed using adhesive film (Eppendorf). PCR amplification and quantification were done in the Bio-Rad CFX 96 Connect $^{\mathrm{TM}}$ detection system (Bio-Rad, Hercules CA, USA). RT-PCR thermocycling was initiated at $50^{\circ} \mathrm{C}$ for $10 \mathrm{~min}$ to synthesize cDNA; this step was followed by $95^{\circ} \mathrm{C}$ for $5 \mathrm{~min}$ followed by 40 cycles of $95^{\circ} \mathrm{C}$ for $10 \mathrm{~s}$ and $60^{\circ} \mathrm{C}$ for $30 \mathrm{~s}$. After the 40 cycles, melting curve analyses from $65^{\circ} \mathrm{C}$ to $95^{\circ} \mathrm{C}$ with $0.05 \mathrm{~s}$ incremental steps of $0.5^{\circ} \mathrm{C}$ was carried out to ensure production of a single DNA product. All tests were run in triplicate.

\subsection{Smartphone-based detection device}

A smartphone-based device was prototyped to analyze the 96-well microplate containing different PCR products after amplification of varying initial concentrations. Figure 1a shows the 96-well microplate and the apparatus to quantitatively detect $E$. coli by monitoring the fluorescence of the PCR reaction using fluorescent dyes for different initial RNA concentrations. The excitation wavelength and the fluorescent emission of SYBR Green I are centered at $497 \mathrm{~nm}$ and $520 \mathrm{~nm}$, respectively, as illustrated in Figure 1b.

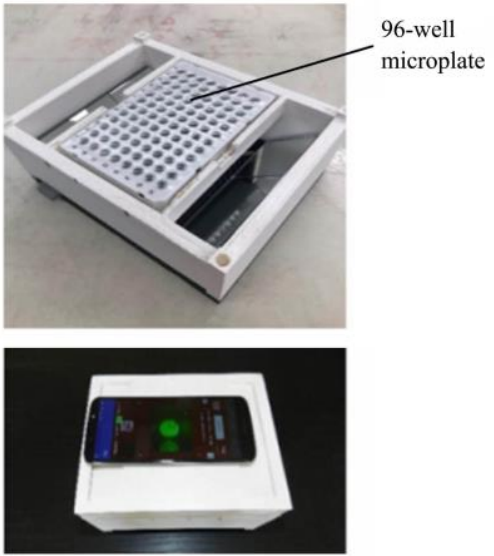

a. Smartphone-based apparatus for detection of $E$. coli

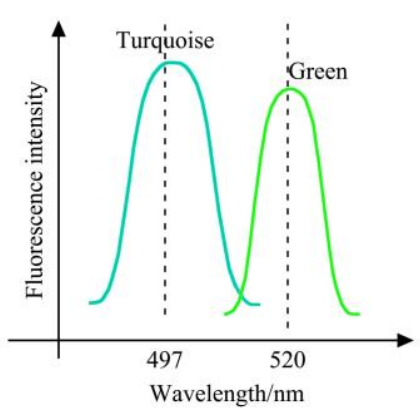

b. Fluorescence spectra of SYBR Green I

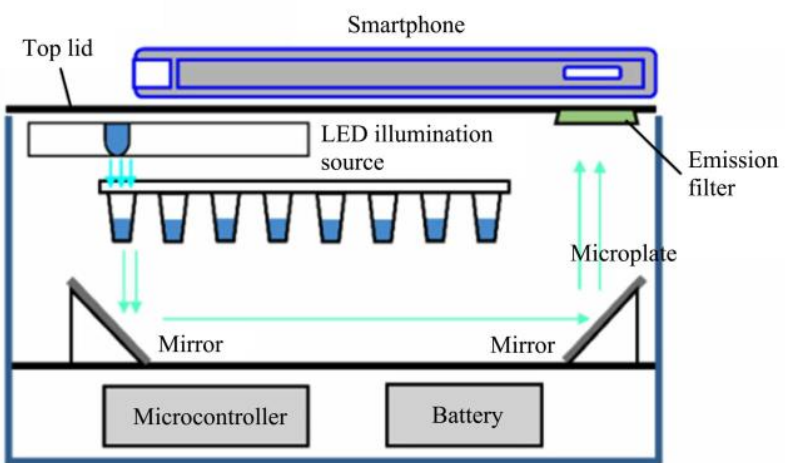

c. Schematic illustration of the overall work design

Figure 1 Experimental apparatus and schematic illustration

Figure 1c depicts a schematic of the overall design for fluorescent detection. The portable apparatus was engineered to adapt to different types of smartphones. In order for the apparatus to be used by any type of phone with different camera positions, the phone is located on the top lid of the apparatus. The 3D-printed box encloses full system assembly, obscuring ambient light and allowing only for LED illumination. The main compartment of the box houses LED light source, the Eppendorf microplate, a bandpass filter, and reflecting mirrors. The apparatus has a power bank installed inside the device that can support the LED, a USB port to recharge the power bank, and a switch control for the LED. The 96-well microplate was placed inside the device to prevent any other light from infiltrating the PCR assays with SYBR Green I. A user adjusts the LED upon 
the designated area of the 96-well microplate so that the LED light source illuminates the specific well of the microplate. Reflective mirrors were set at $45^{\circ}$ to project the image from the sample to the smartphone camera for imaging analysis. To capture the fluorescence image, an optical bandpass filter (equal to 520/10 nm) was used in this study that transmits light only within emission spectra in the green $(515-525 \mathrm{~nm})$ range. The bottom compartment of the apparatus contains electronics, wires, and circuits. The circuit is to power the LED light source and have an auto-shutoff sequence after a duration of $2 \mathrm{~h}$.

\subsection{Smartphone application programming}

A smartphone software application (APP) was specifically developed for this study to process images to quantify the bacterial count. The APP can be used intuitively, requiring minimal input from an untrained user. The APP algorithm for fluorescent signal measurement involves capturing, storing and processing the image of the sample. Color information from the image is extracted and matched with the Red, Green, Blue (RGB) values to assist the user in selecting the target color. The source code of the android APP was created in the Android Studio Java language. The code created different functions in the APP including the User Interface (UI), various buttons, slides, error messages and other visuals available to the user as shown in Figure 2.

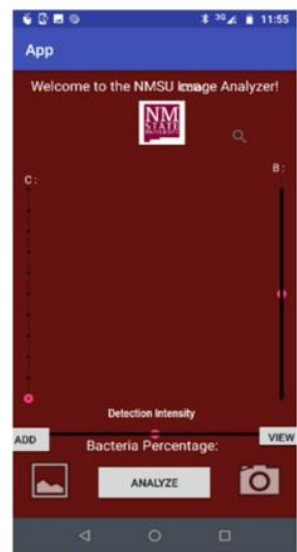

a. Application design using an android smartphone

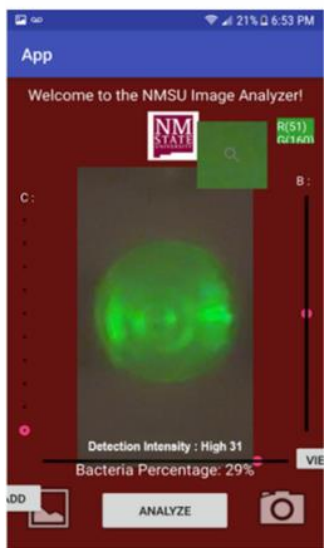

b. Fluorescent image of one of the wells from a 96-well plate captured by a smartphone camera

Figure 2 Smartphone enabled $E$. coli detection

The android APP begins with the main screen which includes three slides and five buttons. Once an image is selected, the user is able to touch a portion of the image and read the RGB value of the selected pixel in the top right corner. Next to that, a zoomed-in view of the selected portion will appear so the user can easily identify where they touched and which pixel is selected. The pixel analysis is processed and the percentage value of matching pixels throughout the entire image, which is correlated with the target nucleic acid concentration, is calculated and displayed by pressing the analyze button. The main function of the application is to allow the user to define the color of the emitted fluorescent light that will be utilized to detect the target within the sample. Once an image is taken, the application allows the user to interact with the selected image and allows for the touched pixel of the image to determine the RGB value. This function assists the user in pinpointing the target pixels and allows for bitmaps to be analyzed in a specific region.

\section{Results and discussion}

\subsection{RT-PCR amplification of $E$. coli}

The extracted RNA was quantified with a NanoDrop ND-1000 spectrophotometer (Thermo Fisher Scientific, Waltham, MA). The dnaK and rpoA primers were used for RT-PCR amplification and products were detected by monitoring the increase in fluorescence by adding SYBR Green I to the PCR reaction mix. Figure 3 shows RT-PCR amplification results with the dnaK primer for 5 initial RNA concentrations where fluorescence intensities (product accumulation) are plotted with respect to the cycle number. A Relative Fluorescence Unit (RFU) is used to indicate the fluorescence intensities detected in Figure 3. SYBR Green I produces a weak fluorescence signal in the presence of single-stranded DNA but emits a strong fluorescence signal upon binding to double-stranded DNA as shown in Figure $3 \mathrm{~b}$. Amplification results confirm that the threshold cycle of the detectable signal is primarily determined by the initial quantity of template at the start of the PCR amplification.

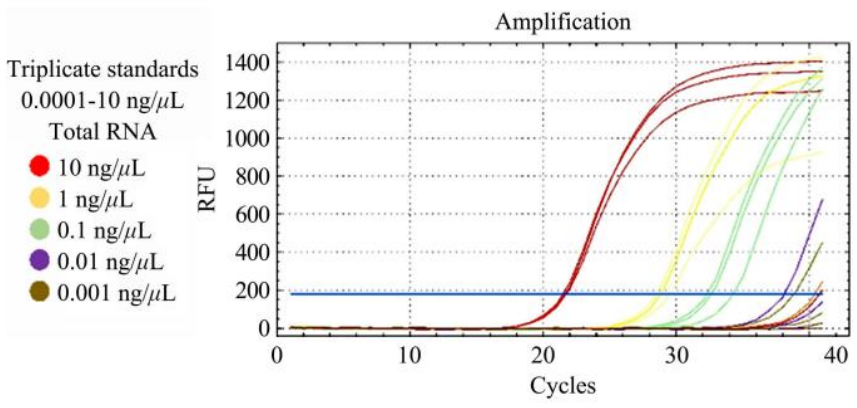

a. Amplification of dnaK products of PCR over time (cycle)
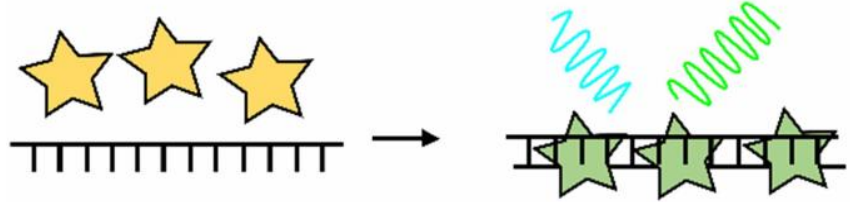

b. Schematic drawing of DNA-intercalating dye SYBR Green I

Figure 3 RT-PCR amplification results

Figure 4 shows the standard curve generated using different sample concentrations with the dnaK primer. The quantification cycle $(\mathrm{Cq})$ values of the set were calculated by Bio-Rad's CFX Connect software. The X's indicate that samples were removed from the curve calculation since they deviated from their triplicate. The linear regression line was generated to correlate quantification cycles (Cq) to copy numbers. The $R^{2}$-value of the linear regression line is 0.934 . Linearity provides a measure of the variability across assay replicates. RT-PCT detection results with the rpoA primer showed higher variability with the $R^{2}$-value of 0.786 , indicating that amplification efficiency varies for different primers.

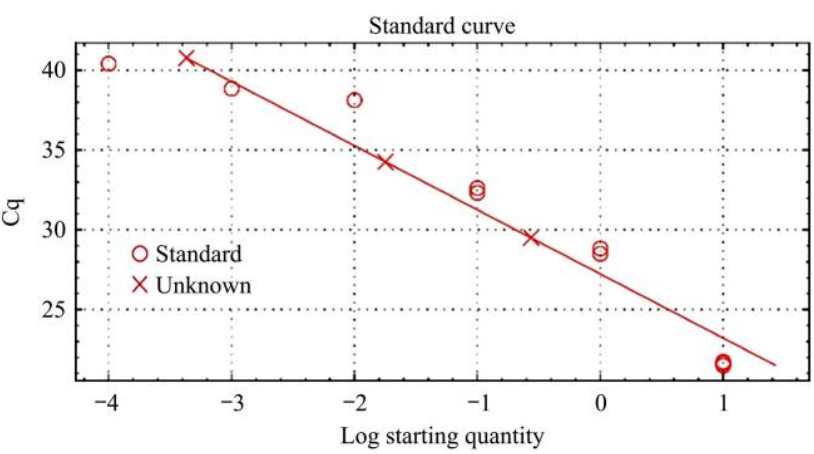

Figure 4 PCR Standard curve at different RNA concentrations $(\mathrm{ng} / \mu \mathrm{L})$ with the $d n a K$ primer

3.2 Mobile application for color sensitivity assay detection The user interface of the application displays the magnified 
view of the small region touched by the user and the RBG values in the top right region of the smartphone screen (Figure $2 b$ ). This function helps the user to select the pixel of the target fluorescent color and in the present experiment, we selected pixels having RGB values of R: 0-51, G: 160-163, B: 7-49, which is the range of values of the emitted signal. Figure $2 b$ shows a typical image of the fluorescent signal taken from a sample. Fluorescent color changes in end point detection can be monitored by human vision. However, a digital camera sensor is more quantitative than the human eye as parameters of human vision such as stereo vision, visible color gamut, dynamic color range, and retinal photon sensitivity vary from eye to eye $\mathrm{e}^{[20,21]}$.

The proposed smartphone detection system analyzed the PCR products whose initial concentrations of template RNA were $10 \mathrm{ng} / \mu \mathrm{L}, 1 \mathrm{ng} / \mu \mathrm{L}, 0.1 \mathrm{ng} / \mu \mathrm{L}$ and $0.01 \mathrm{ng} / \mu \mathrm{L}$, respectively. The APP showed promising testing results of quantifying $E$. coli by the fluorescent dye. The application analyzed the percentage value of selected RGB values of the fluorescent signal compared to all pixels. The application code counts every time it finds a matching pixel that fits within the range that the user specifies. The PCR products were analyzed by two different primers: dnaK and rpoA. Figure 5 shows the average percentages of fluorescence detected in each assay for four initial concentrations of template RNA. It is apparent that the percentage of fluorescence and initial concentration of template RNA have a linear relationship, with $R$-values of linearly related to the PCR reaction products. Utilizing the present fluorescent color analysis technique resulted in comparable accuracy to the real-time PCR fluorescent signal detection.

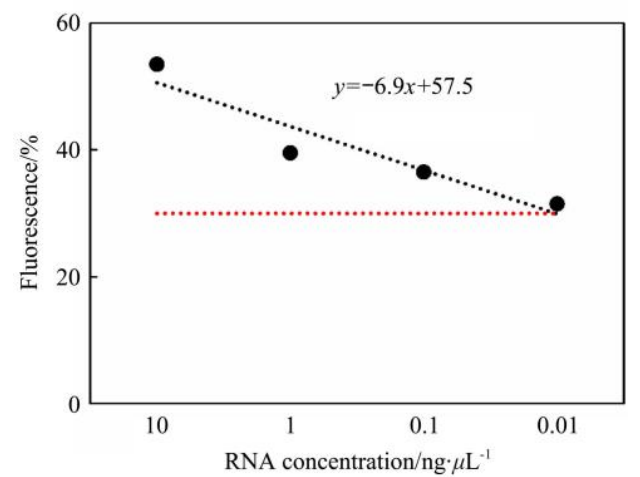

a. dnaK primer (black circle) and non-template control (red dotted line)

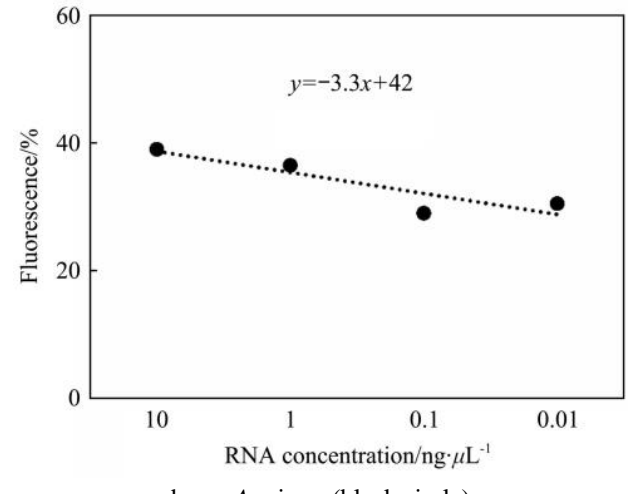

b. rpoA primer (black circle)

Figure 5 Fluorescence percentage $(\%)$ calculated by the smartphone APP vs initial concentration of PCR assay

The correlations between the smartphone fluorescence percentage and RNA concentrations were $R^{2}=0.893$ and 0.797 for the dnaK and rpoA primers, respectively. It is interesting to note that the thermal cycler $R^{2}$ values $\left(R^{2}=0.934\right.$ for $d n a K$ primer and
$R^{2}=0.786$ for rpoA primer) reflected the same quality of non-linearity as the cell phone $R^{2}$ values. The red line in Figure 5 represents non-template control, which shows no significant change in fluorescent percentage. Utilizing the present fluorescent color analysis technique resulted in comparable accuracy to the real-time PCR fluorescent signal detection.

Table 1 RT-PCR Statistical analysis for $d n a K$ and $r p o A$ primers

\begin{tabular}{ccccc}
\hline & $E$ & $R^{2}$ & Slope & $y$-intercept \\
\hline dnaK & $77.3 \%$ & 0.934 & -4.019 & 27.234 \\
rpoA & $158.7 \%$ & 0786 & -2.422 & 28.505 \\
\hline
\end{tabular}

\section{Conclusions}

Smartphone-based detection of $E$. coli was successfully demonstrated with the proposed smartphone-based device. A smartphone application was developed to eliminate the inaccurate monitoring by the human eye. The smartphone APP measured the percentage of fluorescence detected from samples, which was proportional to the concentration of the bacterial nucleic acid. This smartphone application was designed to guide an operator with a user-friendly interface through each step of testing. It is concluded that the key innovation of the present study was to combine efficient image processing encoded into a smartphone application with a low-cost $3 \mathrm{D}$ printed device. Future research will incorporate a LAMP method for amplification of nucleic acid sequences that can be run at a constant temperature in a simple heating block module added to the present device. This method will eliminate the need for the dedicated equipment to operate cyclothermal amplification of standard polymerase chain reaction (PCR)-based methods. In addition, closed-tube amplification reactions allow for the detection of amplified DNAS for both end point and real-time analysis.

\section{Acknowledgements}

This work was supported in part by WorkFoS-Ag Program (Grant No. 2021-67037-34163) and the ALFA-IoT Program (Grant No. 2018-38422-28564) from the USDA National Institute of Food and Agriculture.

\section{[References]}

[1] Zhu X, Wang K, Jin Y, Wang S, Liu X, Zhou P, et al. Multiplexed fluorometric determination for three microRNAs in acute myocardial infarction by using duplex-specific nuclease and $\mathrm{MoS}_{2}$ nanosheets. Mikrochim Acta, 2019; 187(1): 1-15.

[2] Zhu H, Sikora U, Ozcan A A. Quantum dot enabled detection of Escherichia coli using a cell-phone. Analyst, 2012; 137(11): 2541-2544.

[3] Zhu H, Yaglidere O, Su T W, Tseng D, Ozcan A. Cost-effective and compact wide-field fluorescent imaging on a cell-phone. Lab on a Chip, $2011 ; 11: 315-322$

[4] Li C, Liu W, Hu J, Zhang C. A single quantum dot-based nanosensor with multilayer of multiple acceptors for ultrasensitive detection of human alkyladenine DNA glycosylase. Chemical Science, 2019; 10: 8675-8684.

[5] Zhu H, Mavandadi S, Coskun A F, Yaglidere O, Ozcan A. Optofluidic fluorescent imaging cytometry on a cell phone. Analytical Chemistry, 2011; 83: 6641-6647.

[6] Ludwig S K J, Zhu H, Phillips S, Shiledar A, Feng S, Tseng D, et al. Cellphone-based detection platform for rbST biomarker analysis in milk extracts using a microsphere fluorescence immunoassay. Analytical and Bioanalytical Chemistry, 2014; 406: 6857-6866.

[7] Park T S, Yoon J Y. Smartphone detection of Escherichia coli from field water samples on paper microfluidics. IEEE Sensors Journal, 2015; 5: 1902-1907.

[8] Park T S, Li W, McCracken K E, Yoon J Y. Smartphone quantifies 
Salmonella from paper microfluidics. Lab on a Chip, 2013; 13: 4832-4840.

[9] Lee S, Kim G, Moon J. Performance improvement of the one-dot lateral flow immunoassay for aflatoxin B1 by using a smartphone-based reading system. Sensors, 2013; 13: 5109-5116.

[10] Mora C A, Herzog A F, Raso R A, Stark W J. Programmable living material containing reporter micro-organisms permits quantitative detection of oligosaccharides. Biomaterials, 2015; 61: 1-9.

[11] Priye A, Bird S W, Light Y K, Ball C S, Negrete O A, Meagher, R J. Smartphone-based diagnostic platform for rapid detection of Zika, chikungunya, and dengue viruses. Scientific Reports, 2017; 7: 44778. doi: $10.1038 /$ srep44778

[12] Hardinge P, Murray J A H. Reduced false positives and improved reporting of loop-mediated isothermal amplification using quenched fluorescent primers. Scientific Reports, 2019; 9: 7400. doi: 10.1038/ s41598-019-43817-z.

[13] Jang W S, Lim D H, Yoon J, Kim A, Lim M, Nam J, et al. Development of a multiplex Loop-Mediated Isothermal Amplification (LAMP) assay for on-site diagnosis of SARS CoV-2. PLOS ONE, 2021; 16(3): e0248042. doi: 10.1371/journal.pone.0248042.

[14] García-Bernalt Diego J, Fernández-Soto P, Crego-Vicente B, Alonso-Castrillejo B, Febrer-Sendra A, Gómez-Sánchez A, et al. Progress in loop-mediated isothermal amplification assay for detection of Schistosoma mansoni DNA: towards a ready-to-use test. Scientific Reports, 1029; 9: 14744 . doi: 10.1038/s41598-019-51342-2.
[15] BIO RAD. iTaq universal SYBR green one-step kit. Available: http://www.bio-rad.com/webroot/web/pdf/lsr/literature/10032048.pdf. Accessed on [2020-08-15].

[16] Wang B, Duan H, Chong P, Su S, Shan L, Yi D, et al. Systematic selection and validation of suitable reference genes for quantitative real-time PCR normalization studies of gene expression in Nitraria tangutorum. Scientific Reports, 2020; 10(1): 1-10.

[17] Torres S, Lama C, Mantecón L, Flemetakis E, Infante C. Selection and validation of reference genes for quantitative real-time PCR in the green microalgae Tetraselmis chui. PLOS ONE, 2021; 16(1): 1-26.

[18] Castillo H, Schoderbek D, Dulal S, Escobar G, Wood J, Nelson R, et al Stress induction in the bacteria Shewanella oneidensis and Deinococcus radiodurans in response to below-background ionizing radiation. Int. J. Radiat. Biol., 2015; 91(9): 749-756.

[19] Castillo H, Smith G B. Below-background ionizing radiation as an environmental cue for bacteria. Front. Microbiol., 2017; 8: 177. doi: 10.3389/fmicb.2017.00177.

[20] Ruminski D, Palczewska G, Nowakowski M, Zielinska A, Kefalov V, Komar K, et al. Two-photon microperimetry: sensitivity of human photoreceptors to infrared light. Biomedical Optics Express, 2019; 10(9): 4551-4567.

[21] Zhang F, Kurokawa K, Lassoued A, Crowell J A, Miller D T. Cone photoreceptor classification in the living human eye from photostimulationinduced phase dynamics. Proceedings of the National Academy of Sciences of the United States of America, 2019; 116(16): 7951-7956. 